\title{
ON PROPOSITIONAL PLATONISM, REPRESENTATION, AND DIVINE CONCEPTUALISM
}

\author{
C.P. RULOFF \\ Kwantlen Polytechnic University
}

\begin{abstract}
Gould and Davis (2014) have recently argued for the claim that Propositional Platonism is mistaken since it is not able to explain how a proposition comes to bear its representational properties. But, say Gould and Davis, if Propositional Platonism is mistaken, then Divine Conceptualism must be true and we should therefore identify propositions with the contents of a divine mind, i.e., God. In this paper, I argue that Gould and Davis' argument against Propositional Platonism fails since it depends upon a number of assumptions that the Propositional Platonist need not accept.
\end{abstract}

\section{GOULD AND DAVIS’ ARGUMENT}

Call "Propositional Platonism" the view according to which propositions are (minimally) abstract, mind and language-independent, truthbearing entities. In their "Modified Theistic Activism" (2014), Gould and Davis have recently advanced an argument which, if correct, shows that Propositional Platonism is mistaken. Their argument goes as follows. According to Gould and Davis, if Propositional Platonism is true, then propositions must be abstract representational entities; that is, they must be of or about things. And this, say Gould and Davis, is an essential property of propositions, for if propositions failed to be representational, propositions couldn't be about anything, and so bear a truth-value. As Gould and Davis (2014) put it:

In short, propositions ... are intentional objects; they are of or about things. And this is an essential property of propositions; for if they lacked this property, they could not possibly be claims or assertions of any kind, they could not represent anything, in which case they could not be true (/false). (p. 52-53) 
Now, if propositions are abstract representational entities, then, say Gould and Davis, they will be structured entities. For example, if the proposition expressed by (1):

(1) Quine is wise.

is an abstract representational entity, then, say Gould and Davis, the proposition expressed will be a structured entity or $n$-tuple, one that has as its constituent components properties, relations, and concrete individuals. Specifically, if the proposition expressed by (1) is an abstract representational entity, then, say Gould and Davis, it will have as its constituents Quine, the property of wisdom, and the exemplification relation. ${ }^{1}$ As Gould and Davis put it, the proposition expressed by (1) will contain:

an admixture of concrete particulars and abstract objects. On the concrete side of things, (1) would contain Quine himself as a constituent. But its other ingredients would include the (abstract) property of wisdom, along with the (abstract) exemplification relation Quine stands in to that property. (p. 53)

Now, if propositions are structured abstract entities that have as their constituents properties, relations, and concrete individuals, then, say Gould and Davis, propositions must derive their representational properties solely from these constituents. So, since the proposition expressed by (1) contains as constituents Quine, the property of wisdom, and the exemplification relation, (1)'s representational properties must be derived solely from these constituents. As Gould and Davis put it: “... whatever intentionality [(1)] enjoys is inherited or derived; it will be a function of (1)'s parts, each of which is essential to it." (p.53). But, say Gould and Davis, the constituents of the proposition expressed by (1) plainly fail to be representational, for the constituent <Quine $>$ in (1) is not in any way about Quine. But now, say Gould and Davis, if the constituents of the proposition expressed by (1) fail to be representational, then, by extension, the proposition expressed by (1) as a whole fails to be representational as well. As Gould and Davis put it:

Since Quine is not of or about anything, since he does not represent anything (even himself), the mere fact that something contains him will

${ }^{1}$ Hence, the proposition expressed by (1) can be represented as:

(1') Exemplification (Quine, wisdom) 
not make that thing about Quine. You might as well argue that Harvard's philosophy department was about Quine because it contained him as a member. The point is: just in himself, Quine, while impressive in many ways, is an intentional flop. (p. 54)

But now, if the proposition expressed by (1) fails to be representational, it follows, say Gould and Davis, that Propositional Platonism must be mistaken and therfore should be rejected. Formally, the argument that Gould and Davis present against Propositional Platonism goes as follows:

\section{THE GOULD/DAVIS ARGUMENT AGAINST PROPOSITIONAL PLATONISM}

(P1) If Propositional Platonism is true, then propositions are representational, truth-bearing entities.

(P2) If propositions are representational, truth-bearing entities, then propositions are structured entities.

(P3) If propositions are structured entities, then propositions have as their constituents properties, relations, and concrete individuals.

(P4) If propositions have as their constituents properties, relations, and concrete individuals, then the representational properties of a proposition (and by extension, its truth-conditions) must be explained solely in terms of these constituents.

(P5) It's not possible to explain the representational properties of a proposition (along with its truth-conditions) in terms of these constituents.

(P6) Therefore, Propositional Platonism is mistaken.

Gould and Davis go on claim that, since (P6) is true and Propositional Platonism doesn't contain the conceptual resources to explain the representational properties of propositions whereas Divine Conceptualism does contain such resources, we should reject Propositional Platonism in favor of Divine Conceptualism and identify abstract propositions with the contents of a divine mind, i.e., God.

Gould and Davis' argument - which has recently received critical attention from Craig (2014), Welty (2014), Yandell (2014), Shalkowski (2014), and Oppy (2014) - is deductively valid and, if sound, amounts to a wholesale refutation of Propositional Platonism. In what follows, 
however, I want to argue that Gould and Davis' argument against Propositional Platonism (hereafter "Platonism") depends upon at least five assumptions that the Platonist need not accept. If I'm right about this, then Gould and Davis' argument against Platonism fails and that, accordingly, we need not endorse Divine Conceptualism and identify abstract propositions with the contents of a divine mind. ${ }^{2}$

\section{2.a. Gould and Davis' First Assumption}

The first controversial assumption upon which Gould and Davis' argument depends is this:

(a1) On Propositional Platonism, propositions must be representational in order to be the bearers of truth-values.

Gould and Davis apparently think that (a1) is somehow self-evident or obviously true. Indeed, Gould and Davis go so far as to claim that being representational is an "essential" property of abstract propositions. But is (a1) even true? Recent work by Speaks (2014) indicates that (a1) is in fact false and that the Platonist need not be committed to the claim that propositions must be representational in order to be the bearers of truthvalues. Glossing over details, Speaks' account goes as follows. Consider the monadic predication:

(2) Smith talks

Call the proposition expressed by (2) PROP, and suppose that the content of "Smith" is a certain person, Smith, and the content of "talks" is the monadic property of talking. (In this case, we can call Smith and the property of talking the constituents of PROP.) So what is PROP, according to Speaks? According to Speaks, PROP is a property. That is, on Speaks' account, the proposition expressed by (2) just is the property of being such that Smith instantiates talking. And, according to Speaks, PROP is true provided that the property of being such that Smith instantiates talking is in fact instantiated. Generalizing, on Speaks' account, propositions are properties which are true iff those properties are instantiated; on Speaks' account, "propositions are true with respect to a world $\mathrm{w}$ iff were $\mathrm{w}$ actual, that property would be instantiated" (2014, p.76).

\footnotetext{
${ }^{2}$ I should note that the objections that I develop in this paper are, so far as I can tell, logically independent of those advanced by Craig, Welty, Yandell, Shalkowski, and Oppy.
} 
According to Speaks, viewing propositions as properties fits with much of our ordinary talk about propositions. We might say, says Speaks, that believing a proposition is taking the world to be a certain way. But if "ways things are" are properties, this indicates that having a belief is taking a certain propositional attitude toward a property; if "ways things are" are properties, then the objects of mental states and speech acts are themselves properties.

What is central for our purposes is this: On Speaks' account, propositions (construed as properties) have truth-conditions, and are abstract, but they are not about anything - propositions are not representational entities. Beliefs, assertions, and so on, says Speaks, have truth-conditions and are representational, and they are representational because of their relations to propositions; but propositions themselves are not representational entities. They are simply entities that are true or false, and fundamentally so. Speaks' view, then, does away with the traditional idea that a proposition must be a representational entity, i.e., an entity that represents things as they really are. Indeed, according to Speaks, to say that something is true just in case it represents the world as being some way is a mere platitude, and a platitude that ought to be given up, for who, says Speaks, says the platitude must apply to everything that is true or false? Says Speaks (2014, p. 221):

On my view, it is a platitude that a sentence is true iff it represents the world as being some way, and the world is that way - what it is for a sentence to represent the world as being some way is for that sentence to have a certain property - a way things could be - as its content, and what it is for the world to be that way is for the property to be instantiated. But no such claim holds about the truth of propositions. ${ }^{3}$

Doing away with the idea that propositions are representational entities may seem like a cost. But, as Speaks stresses, there is also a real benefit here; since the idea that an abstract entity can be representational has seemed to many to be a bizarre one, "if we can give an account of truth ... without making use of entities of this sort, this is a good thing" (Speaks, 2014, p. 78).

The salient point here is this: Gould and Davis assume that (a1) is obviously true and that the Platonist is somehow committed to the claim that propositions must be representational in order to be the bearers

\footnotetext{
${ }^{3}$ Italics added.
} 
of truth-values. But, if Speaks' account is correct, then the Platonist is not so committed, for, on Speaks' account, propositions are abstract, non-representational truth-bearing entities. Since Gould and Davis fail to provide any reasons for thinking that Speaks' account is in any way mistaken or not worthy of serious consideration - indeed, since Gould and Davis fail to even consider how Speaks account undercuts the truth of (a1) - we must conclude that (a1) is false, or, at the very least, not obviously true. ${ }^{4}$

\section{2.b. Gould and Davis' Second Assumption}

The second controversial assumption upon which Gould and Davis' argument depends is this:

(a2) On Propositional Platonism, propositions are abstract entities whose constituent components are properties, relations, and concrete individuals; on Platonism, propositions are to be given a specifically Russellian analysis.

To be sure, Platonists often claim that a Russellian analysis of propositions is correct and that relations, properties, and concrete individuals, serve as the constituent components of propositions. But is the Platonist somehow committed to a Russellian analysis, as Gould and Davis' (a2) indicates? Contrary to Gould and Davis' (a2), there is in fact a wide range of non-Russellian structured analyses of propositions available to the Platonist.

For instance, the main alternative to the Russellian account is the broadly Fregean account according to which propositions are structured abstract entities that have as their constituent components, not relations, properties and concrete individuals, but rather senses. More fully, Frege held that simple linguistic expressions such as proper names have a sense (or "Sinn"), where the sense of a linguistic expression is the thing or object in the world that the expression serves to pick out; to use Fregean terminology, the sense of a linguistic expression is the mode of presentation that determines the referent of that linguistic

\footnotetext{
${ }^{4}$ Let me stress here that I am not claiming that Speaks' account is true. Rather, I am merely claiming that, by failing to consider Speaks' account, Gould and Davis imply that it's somehow obvious that propositions must be representational in order to be truthvalue bearers. But, if Speaks' account is corect, then, contrary to Gould and Davis, it's not at all obvious that propositions must be representational in order to be truth-value bearers.
} 
expression. ${ }^{5}$ Frege extends this analysis to complex linguistic expressions such as propositions as well. According to Frege a complete declarative sentence also possesses a sense, where the sense of a declarative sentence is a proposition, or in Frege's words, a "thought." Moreover, and most importantly for our purposes, on the Fregean account, the proposition associated with or expressed by a declarative sentence is determined wholly by the senses of its subsentential components and of how those components are syntactically arranged. As Burge puts it, (2005), on what we might call Fregean Semantic Atomism, the meaning or sense of a complex expression is "functionally dependent only on the senses of its logically relevant component expressions" (p.85). Or, as King (2011) puts it, on the Fregean account, "the proposition/thought expressed by a sentence is a function of the senses of the words in the sentence and how they are put together." The salient point here, then, is this: Fregeans agree with Russellians that propositions are structured abstract entities, but hold that the constituents of propositions are not objects, properties, and relations, but rather senses or modes of presentation. ${ }^{6}$

An altogether different structured account is the broadly Mooreaninspired structured-concepts account provided by Swartz and Bradley (1979, pp. 87-97). Very roughly, according to the Swartz/Bradley account, propositions are structured abstract entities whose constituent components are ordered concepts. ${ }^{7}$ For example, on the Swartz/Bradley analysis, the constituents of the proposition expressed by:

(4) Muhammad Ali is an Olympic skier

are the contingently applicable concepts of being Muhammad Ali and being an Olympic skier, where a concept is, roughly speaking, an open sentence which contains a gap such that, when the gap is filled with an appropriate expression, the resulting sentence expresses something

${ }^{5}$ So, for example, the sense of the proper name "Barack Obama" is, for Frege, the entity in the world that the name "Barack Obama" picks out or stands for. Here one might think of the sense of the name "Barack Obama" as some identifying descriptive content that serves to uniquely pick out the person Barack Obama.

${ }^{6}$ Note: on the Fregean account, senses are abstract objects.

${ }^{7}$ Where concepts are expressible by those words which feature in a kind of open sentence. An open sentence in general is a sentence which contains a gap such that when the gap is filled with an appropriate expression, the resulting closed sentence expresses something that is true or false. See Swartz and Bradley (1979) pages 88-89 for more on concepts. 
that is true or false. ${ }^{8}$ Hence, on the Swartz/Bradley analysis, the conceptual constituents of the proposition expressed by (4) are the members of the set:

\{being Muhammad Ali, being an Olympic skier\}

which may be expressed by saying:

the item of which being Muhammad Ali is the concept has the attribute of which being an Olympic skier is the concept.

or, even more formally:

there is an item $\mathrm{x}$ such that $\mathrm{x}$ falls under the concept of being Muhammad Ali and $\mathrm{x}$ falls under the concept of being an Olympic skier.

Generalizing, Swartz and Bradley write that, if we let the Strawsonian locution:

“ass $\{\ldots\}$

represent the idea which assigns attributes of which certain constituents are the concepts to items of which other constituents are the concepts, we can say that the structure:

ass $\{$ being Muhammad Ali, being an Olympic skier\}

just is the proposition expressed by (4). On the Swartz/Bradley analysis, then, a proposition just is its constituent concepts standing in the proposition-yielding relation. ${ }^{9}$ The salient point here, then, is this: Swartz and Bradley agree with Russellians that propositions are structured abstract entities, but hold that the constituents of propositions are not objects, properties, and relations, but rather ordered concepts. And of course, there are other well-known structured accounts. ${ }^{10}$

8 The particular kind of open sentence which can express a concept is that in which the gap is to be filled either (1) by a referring expression of some kind or (2) by a sentence expressing a proposition. So, for instance, the open sentence: “... is an Olympic skier" is a concept-expressing sentence, on the Swartz/Bradley analysis.

${ }^{9}$ Note: Concepts are, along with Fregean senses, abstract objects.

${ }^{10}$ There is Lewis and Cresswell's structured intensions approach, where propositions are identified with structured intensions; there is Zalta and Menzel's algebraic approach where propositions are identified with zero-place relations. And there are other analyses still. 
Generalizing, the worry here is this: according to Gould and Davis' (a2), the Platonist is somehow committed to the claim that propositions are structured abstract entities that have as their constituent components properties, relations, and concrete individuals. ${ }^{11}$ But this assumption, as we've just seen, is manifestly false, for there is in fact a wide range of nonRussellian structured analyses available to the Platonist. Since Gould and Davis fail to provide any reasons for thinking that any of these competing analyses are mistaken or not worthy of serious consideration - indeed, since Gould and Davis fail to even consider any of the main alternatives to the Russellian account along with the contemporary defenses of these accounts - we must conclude that Gould and Davis' (a2) is false.

\section{2.c. Gould and Davis' Third Assumption.}

The third controversial assumption upon which Gould and Davis' argument depends is this:

(a3) On Propositional Platonism, propositions are structured abstract entities.

But we might question whether the Platonist is even committed to the structured account of propositions in the first place. To be sure, the structured account is the dominant account of propositions; but it is a mistake to assume, as Gould and Davis' (a3) indicates, that the Platonist is somehow committed to such an account for, as a number of theorists have pointed out, the Platonist is free to eschew the structured account altogether and construe propositions as unstructured abstract entities. For instance, according to Bealer's (1993) seminal work on propositions, propositions are unstructured, ontologically primitive sorts of abstracta. More fully, Bealer associates with each proposition a decomposition tree, where such a tree highlights "which logical operations on which entities (individuals, properties, relations) a given proposition is the result of" (Bealer in Carrara and Sacchi, 2006, p. 20). By showing which logical operations a given proposition is the result of, Bealer is able to provide a detailed algebraic structure to propositions. However, and this deserves underscoring, although Bealer is able to provide a detailed

${ }^{11}$ At the very least, since Gould and Davis confine their attention solely to the Russellian analysis, (a2) indicates that the Russellian analysis is somehow the only serious or viable structured analysis of propositions and that any alternative analysis can be safely ignored. 
algebraic structure to propositions, Bealer denies that propositions have constituent components. This is because, as I just indicated, Bealer holds that propositions are metaphysically simple abstract entities that contain no constituents whatsoever (Carrara, and Sacchi, 2006, p. 20). If Bealer's so-called "primitive entity" account of propositions is right, then, although it's possible to attribute a structure to propositions, propositions are ultimately irreducible to their constituents.

An alternative unstructured account of propositions is the possible worlds account according to which propositions are identified with sets of metaphysically possible worlds. More fully, some Platonists have identified each proposition with the set of possible worlds in which that proposition is true, or identified propositions with functions from possible worlds to truth-values. As Stalnaker puts it: (2008): “...it seems reasonable to use sets of possible worlds, or (equivalently) functions from possible worlds into truth-values, to play the role of propositions in our theory" (p.148). So, for example, the proposition expressed by the sentence:

(5) Bill sleeps.

just is, according to the possible worlds account, the set of worlds in which the referent of $<$ Bill $>$ is a member of the set of things that sleep, or, equivalently, a function $f$ that maps a possible world $w$ to the value True iff Bill sleeps in $w$. Now, although sentence (5) is structured and each part of the sentence is of a determinate semantic type (an individual constant and a monadic property), the proposition expressed by (5) is not structurally isomorphic to sentence (5), for a set of possible worlds is not a linguistically structured entity at all (Collins, 2011, p. 8).

Indeed, it is worth mentioning that the Platonist possesses reasons for being skeptical of the structured account of propositions in the first place. Here's why; It is widely held that the semantic value or meaning of a complex expression is determined by the semantic values of its constituents and the way in which that those constituents are structurally arranged. Formally, according to the so-called Principle of Compositionality (PC):

PC: The semantic value of a complex expression $e$ is composed out of the semantic values of $e$ 's constituent components and the way in which these components are structurally arranged. 
Now if, as Keller and Keller (2014) have recently noted, PC is in fact true, then the structured account of propositions along with Gould and Davis (a3) immediately follows. Keller and Keller's argument proceeds as follows. Suppose that PC is true and that the semantic value of a complex expression is composed out of the semantic values of its components and the way in which these components are structurally arranged. But, since propositions just are the semantic values of complex expressions, it follows that a proposition is composed out of the semantic values of a complex expression's components and the way in which these components are structurally arranged, and so is a structured entity. Formally, Keller and Keller's argument goes as follows:

\section{The Compositionality Argument}

(1) PC: The semantic value of a complex expression $e$ is composed out of the semantic values of $e$ 's components and the way in which these components are structurally arranged.

(2) A proposition is the semantic value of a complex expression $e$.

(3) Hence, a proposition is composed out of the semantic values of $e$ 's components and the way in which e's components are structurally arranged, and thus is a structured entity.

It's important to note that this argument is typically cited as the main reason for adopting the structured account. Since the argument is deductively valid and since (2) is not in dispute, the acceptability of this argument hinges entirely upon premise (1). Proponents of the structured account support (1) by pointing to the productivity, understandability, and systematicity of language, and argue that (1) is the best, or only, way to explain these features. ${ }^{12}$ Recent work by Keller and Keller (2014), however, strongly indicates that (1) is in fact false; Keller and Keller claim that (1) is either subject to a range of counterexamples or depends upon a number of highly controversial assumptions. But now, if (1) is mistaken and (1) is the main reason for adopting the structured account, then, say

12 According to Keller and Keller, a representational system $L$ is productive "just in case finite beings can use $L$ to produce an infinite number of meaningful complex expressions; it is understandable just in case someone competent with $L$ (i.e., who grasps the finite lexicon and grammatical rules of $L$ ) is capable of understanding complex expressions of $L$ she has never before encountered; and it is systematic just in case whenever someone competent with $L$ is capable of understanding an expression $e$ of $L$, she is capable of understanding systematic variants of $e$ (expressionsobtained by permuting the constituents of $e$ )". 
Keller and Keller, the fundamental rationale for adopting the structured account of propositions is radically undercut. But now, if the fundamental rationale for adopting the structured account is undercut, then Gould and Davis' (a3) is also thereby undercut, and thus, the Propositional Platonist possesses reasons for rejecting the structured account.

The point here is this; according to Gould and Davis' assumption (a3), the Platonist is somehow committed to the claim that propositions are structured abstract entities. But this assumption is false, for, as we've just seen, the Platonist is free to forego the structured account altogether and construe propositions as atomic, unstructured abstract entities. Moreover, if, as I just indicated, the arguments of Keller and Keller against the Principle of Compositionality are correct, then the Platonist possesses an undercutting defeater for (a3), i.e., a reason for thinking that the basic grounds for adopting the structured account of propositions are inadequate. Since Gould and Davis fail to provide any reasons for thinking that the unstructured approach to propositions or the arguments of Keller and Keller against the structured account are in way mistaken, the Propositional Platonist is justified in rejecting (a3). ${ }^{13}$

\section{2.d. Gould and Davis' Fourth Assumption}

The fourth controversial assumption upon which Gould and Davis' argument depends is this:

(a4) On Propositional Platonism, a proposition's truth-conditions must be explained solely in terms of the representational properties of its constituent components.

So, on Gould and Davis' (a4), the truth-conditions of a proposition is to be explained solely in terms of the representational properties of its

${ }^{13}$ To be fair, Gould and Davis do briefly consider the view according to which propositions are "simple, brutely intentional Platonic" Forms, thus implying something like the unstructured account. But Gould and Davis quickly dismiss this view on the grounds that such a view "hardly squares with what we all learned at Socrates' knee, viz., the Forms are neither "propositional" (p.55) nor "representational" (p.55). By way of response: firstly, Gould and Davis here fail to provide an argument for the claim that unstructured propositions - "the Forms", as Gould and Davis call it - cannot be representational. And secondly, even if one were to grant Gould and Davis the claim that the Forms are neither propositional nor representational, it hardly follows that propositions on Bealer's account or the possible worlds account or some alternative unstructured account are neither propositional nor representational. 
constituent components; on Gould and Davis' (a4), the truth-conditions of a proposition must built up from the representational properties of its constituent components.

Briefly, there are at least three lines of response to (a4) available to the Platonist, each of which undercuts (a4). Firstly, note that if Speaks' account of propositions sketched earlier is at all correct, then (a4) is false. Specifically, ifSpeaks' account is correct and a proposition is a property that bears the truth-value True iff that property is instantiated, then, contrary to Gould and Davis' (a4), the Platonist can account for a proposition's being true without even appealing to the idea of representation.

Secondly, there is no reason to suppose that the Platonist is committed to anything like (a4) for, as we saw in Section 2.c., the Platonist can explain the truth-conditions of a proposition wholly in terms of an unstructured account of propositions - say, the possible worlds account. So, to recall an earlier example, the proposition expressed by the sentence:

(5) Bill sleeps.

just is, on the possible worlds account, a function $f$ that maps a possible world $w$ to the value True iff Bill sleeps in $w$; the proposition expressed by (5) just is, on the possible worlds account, the set of possible worlds at which the proposition expressed by (5) is true. Now, although sentence (5) is linguistically structured, the proposition expressed by (5) isn't structurally isomorphic to sentence (5) for a set of possible worlds isn't a linguistically structured entity in the first place (Collins, 2011, p. 8). Hence, contrary to Gould and Davis' (a4), it's possible to explain the truth-conditions of a proposition wholly in terms of an unstructured account of propositions.

Thirdly, note that if the arguments of Keller and Keller against the Compositionality Argument discussed in the previous section are correct, then the Propositional Platonist possesses an undercutting defeater for the claim that propositions are structured entities that contain constituents. But if the Platonist possesses a defeater for the claim that propositions contain constituents, then the Platonist thereby possesses an undercutting defeater for Gould and Davis' (a4) - for the claim that a proposition's truth-conditions should be explained in terms of its constituent components - for, according to the arguments of Keller and Keller, there are no good reasons for supposing that propositions contain constituent components in the first place. 
The point here is this; on Gould and Davis' (a4), the Platonist must explain the truth-conditions of a proposition wholly in terms of the representational properties of its constituent components. But this, as we've just seen, is false, for, firstly, if Speaks' account of propositions is right, then the Platonist can account for a proposition's being true without even appealing to the idea of representation; secondly, the Propositional Platonist can explain the truth-conditions of a proposition wholly in terms of the unstructured possible worlds account; and lastly, if the arguments of Keller and Keller are correct, then the Platonist possesses an undercutting defeater for the claim that propositions even contain constituent components. Since Gould and Davis fail to provide any reasons for thinking that these responses are mistaken - indeed, since Gould and Davis fail to even consider these potential responses to (a4) - we must conclude that (a4) is false or not obviously true.

\section{2.e. Gould and Davis' Fifth Assumption}

The fifth and most important assumption upon which Gould and Davis' argument rests is this:

(a5) Since the Propositional Platonist isn't able to explain the representational properties of a proposition in terms of its constituent components, a wholesale rejection of Propositional Platonism is justified.

Let's follow Gould and Davis and suppose that the Platonist is in fact committed to the claim that propositions are abstract, structured, representational, truth-bearing entities, and that the representational properties of propositions (so-construed) must be wholly derived from their constituents (where the constituents are properties, concrete objects, and relations). And let's also suppose (along with Gould and Davis) that the Platonist is not in any way able to explain how the representational properties of a proposition is derived from its constituents; that is, let's suppose that the Propositional Platonist is not able to explain the relation of representation. We might now ask: does it follow from these two assumptions that a wholesale rejection of Platonism is justified, as Gould and Davis' (a5) indicates? I don't think it does follow. A number of Platonists have responded to (a5) by claiming something along the following lines: even if the Platonist were to concede that no clear answers are forthcoming as to how an abstract proposition manages to 
derive its representational properties from its constituent components, Platonism is (all things considered) still rationally justified since construing propositions as abstract, mind and language-independent, truth-bearing, representational entities performs a range of explanatory work that no competing account of propositions can. Specifically, the Platonist will claim that, even if she isn't able to explain how an abstract proposition comes to bear the relation of representation, her commitment to Platonism is still, all things considered, rationally justified since Platonism:

- provides a plausible explanation of how the same semantic-content can be expressed by different people uttering different sentencetokens of different languages;

- provides a plausible explanation of how the same semantic-content can be believed by different people;

- provides a plausible account of how mental states gain their representational content;

- provides a plausible account of alethic modality - of necessity, possibility, contingency, and so on.

- makes intuitive sense of our ascriptions of truth and falsity.

And so on. In short, since Platonism elegantly and powerfully simplifies, unifies, and systematizes our thinking about language, thought, and communication, a commitment to Propositional Platonism is warranted even if no explanation is forthcoming as to how an abstract proposition manages to bear its representational properties independently of all minds and language.

To some it may seem mysterious, odd, or even bizarre as to how an abstract proposition can bear its representational properties independently of all minds and language. And perhaps it is mysterious as to how an abstract proposition manages to do this. But it scarcely follows from this that a wholesale rejection of Platonism is justified or warranted, as Gould and Davis's (a5) indicates. Since Platonism is an "impressively powerful account of language, thought, and communication" (Jubien, 1993, p. 103), and since there is (at present) no comparatively powerful rival account of propositions, it is rationally justified to accept Platonism even if the relation of representation remains a completely mysterious one. As Jubien (1993) bluntly puts it, since the overall case for Platonism is so impressively strong, we ought to "try to get used to the mystery" 
(p. 103) of representation. If this line of reasoning is correct, then perhaps the Platonist should ultimately claim that the relation of representation functions as one of the primitive terms in her overall, though presently incomplete, theory of propositions.

The claim that the relation of representation ought to function as a primitive term within the Platonist's overall theory of propositions has frequently been made and defended in the literature. And, as I just pointed out, the relation of representation is not a primitive chosen ad hoc, but a relation that is needed specifically for explanatory purposes. But now, if the Platonist is justified in claiming that the relation of representation functions as a primitive within her overall theory of propositions, then, it seems, Gould and Davis need to provide an argument for the claim that the Platonist is not so justified. But, surprisingly, Gould and Davis fail to provide just such an argument. Indeed, the idea that the relation of representation might function as a primitive within the Platonist's overall theory of propositions is not even considered by Gould and Davis. Since Gould and Davis fail to provide an argument for the claim that the Platonist is not justified in claiming that the relation of representation functions as a primitive in her overall theory of propositions, we then possess a reason for thinking that (a5) is false or not obviously true.

\section{CONCLUSION}

According to Gould and Davis, the Platonist is committed to assumptions (a1)-(a5). But, if the foregoing is correct, then the Platonist need not be committed to any one of (a1)-(a5). The Propositional Platonist need not be committed to the claim that propositions must be representational in order to be the bearers of truth-values; to the claim that propositions must be given a Russellian analysis; to the claim that propositions must be structured abstract entities; to the claim that the representational properties of propositions must be explained in terms of their constituents; to the claim that, since the Propositional Platonism isn't able to explain the relation of representation, Propositional Platonism itself should be rejected. Since the Propositional Platonist need not be committed to any one of (a1)-(a5), we thereby have good reasons for thinking that Gould and Davis' argument against Propositional Platonism fails and thus, by extension, that we need not identify abstract propositions with the contents of a divine mind. Put differently, since Gould and Davis 
fail to engage with the relevant literature that directly challenges the range of assumptions upon which their anti-Platonist argument rests, we must conclude that Gould and Davis' argument against Propositional Platonism does not succeed, and therefore that the inference to Divine Conceptualism is blocked. ${ }^{14}$

\section{BIBLIOGRAPHY}

Bealer, George (1993) "A Solution to Frege's Puzzle" in Philosophical Perspectives, 7, Language and Logic (pp. 17-60). Ridgeview Publishing Company.

Burge, Tyler (2005) Truth, Thought, Reason: Essays on Frege. Oxford University Press.

Carrara, Massimiliano and Sacchi, Elisabetta (2006) Propositions: Semantic and Ontological Issues. Rodopi.

Collins, John (2011) The Unity of Linguistic Meaning. Oxford University Press.

Craig, William Lane, (2014) "Response to Paul M. Gould and Richard Brian Davis" in Gould, Paul, Beyond the Control of God? (pp. 68-70). Bloomsbury Academic.

Gould, Paul and Davis, Richard Brian (2014) "Modified Theistic Activism" in Gould, Paul, Beyond the Control of God? (pp. 51-64). Bloomsbury Academic. Jubien, Michael (1993) Ontology, Modality and the Fallacy of Reference. Cambridge University Press.

Keller, Lorraine and Keller, John (2013) "Compositionality and Structured Propositions". Thought (2): 313-323

King, Jeffrey (2011) "Structured Propositions" in Stanford Encyclopedia of Philosophy. Retrieved from: http://plato.stanford.edu/entries/propositionsstructured/

Oppy, Graham (2014) "Response to Paul M. Gould and Richard Brian Davis" in Gould, Paul, Beyond the Control of God? (pp. 102-104). Bloomsbury Academic.

Speaks, Jeff (2014) "Representation and Structure in the Theory of Propositions" in King, Jeffrey, Soames, Scott, and Speaks, Jeff, New Thinking About Propositions (pp. 215-225). Oxford University Press.

Shalkowski, Scott, (2014) "Response to Paul M. Gould and Richard Brian Davis" in Gould, Paul, Beyond the Control of God? (pp. 66-68). Bloomsbury Academic.

${ }^{14}$ Thanks to Graham Oppy, Patrick Findler, Doran Smolkin, Melinda Hogan, Matthew Davidson, and Stephen Davis for helpful comments and suggestions. 
Swartz, Norman and Bradley, Raymond (1979) Possible Worlds. Hackett.

Stalnaker, Robert (2008) "Assertion" in Portner, Paul and Partee, Barbara, Formal Semantics (pp. 147-161). Wiley Publishing.

Welty, Greg, (2014) "Response to Paul M. Gould and Richard Brian Davis" in

Gould, Paul, Beyond the Control of God? (pp. 66-68). Bloomsbury Academic.

Yandell, Keith, (2014) "Response to Paul M. Gould and Richard Brian Davis" in

Gould, Paul, Beyond the Control of God? (pp. 97-99). Bloomsbury Academic. 